UCRHEP-T187

May 1997

\title{
Seeding of Strange Matter with New Physics
}

\author{
Ernest Ma \\ Department of Physics \\ University of California \\ Riverside, California 92521, USA
}

\begin{abstract}
At greater than nuclear densities, matter may convert into a mixture of nucleons, hyperons, dibaryons, and strangelets, thus facilitating the formation of strange matter even before the onset of the quark-matter phase transition. From a nonstrange dibaryon condensate, it may even be possible to leapfrog into strange matter with a certain new interaction, represented by an effective six-quark operator which is phenomenologically unconstrained.
\end{abstract}

\footnotetext{
${ }^{1}$ Talk given at the International Symposium on "Strangeness in Quark Matter 1997" (Santorini, Greece) April, 1997, to be published in J. Phys. G
} 


\section{Introduction}

The various forms of matter which may exist depend on external conditions such as temperature and pressure. Our knowledge of particles and nuclei comes mainly from experiments near zero temperature and zero pressure, although current and future heavy-ion colliders will probe further into these regimes. A very important question to be answered is whether strange quark matter [1, 2] is stable or metastable under ordinary conditions. We know that at zero pressure, two-flavor $(u$ and $d$ ) quark matter must be highly unstable, or else we would not have stable nuclei. We also know that particles containing strangeness (hyperons) are unstable and decay weakly. However, it is still possible for strange quark matter (containing $u, d$, and $s$ quarks) to be stable or metastable, and be undetected so far because it is not easy to make. After all, iron is the most stable of nuclei, but it only exists because it is made under extreme conditions in the interior of ordinary stars. A possible scenario for strange quark matter is that it is made inside massive neutron stars where the core densities are high enough to pass through the quark-matter phase transition. It has also been suggested[3] that some neutron stars in low-mass x-ray binaries can accrete sufficient mass to undergo a phase transition to become strange stars, for a possible explanation of the observed $\gamma$-ray bursters.

In the following, I propose the possibility that some forms of strange matter are already present inside neutron stars at densities far below that of the quark-matter phase transition. This seeding of strange matter may be possible just from a proper treatment of the physical conditions for stability and equilibrium at high pressure, or more speculatively from a new interaction, represented by an effective six-quark operator which is phenomenologically unconstrained. 


\section{Strangeness Content of Neutron Stars}

It has been known[4] for a long time that at two to three times nuclear density, hyperons such as $\Lambda$ and $\Sigma$ particles may appear inside neutron stars. At last year's Strangeness meeting in Budapest, two detailed analyses were presented [5, 6]. To obtain these results, one must first know that hyperons exist and then have a model describing their interactions with nucleons, electrons, and neutrinos, etc. Suppose one does not know about hyperons, but instead there is evidence for (nonstrange) dibaryons, then a model with dibaryons should be considered inside neutron stars. Just such a possibility was recently considered[7] and the conclusion was that a Bose condensate of dibaryons can occur in nuclear matter before the quark-hadron phase transition.

The lesson we learned from the above is that if we do not know the proper degrees of freedom relevant to the physical conditions of the external environment, then we cannot hope to arrive at the correct conclusion in that particular case. There are things that we do know, such as the existence of hyperons and how they interact under laboratory conditions, but there are also many things that we do not know, such as whether dibaryons are stable or metastable or not, and perhaps more importantly, whether they can be stable in a highpressure environment. Our ignorance by itself cannot justify their omission in a full analysis of what may happen in a neutron star. It would be very interesting to include both hyperons and dibaryons, and perhaps even some strangelets, and postulate interactions among them such as

$$
\begin{aligned}
\Lambda \Lambda & \rightarrow H(A=2, S=-2), \\
H H & \rightarrow T(A=4, S=-4), \\
H T & \rightarrow S^{*}(A=6, S=-6),
\end{aligned}
$$

where $H$ is the $(u d s)^{2}$ dibaryon [8], and $S^{*}$ is the quark-alpha particle [9]. It is important to 
realize that even if the $H, T$, and $S^{*}$ particles do not exist at zero pressure, their dynamics may be such that the above processes have to be included. These quark-alphas should then be locally stable 10 and may coalesce into bulk strange matter within this environment.

\section{New $\Delta \mathrm{S}=3$ Interaction}

If only nonstrange dibaryons form, then a nudge from some new physics may be advantageous for the formation of strange matter. This kind of speculation may or may not be valid depending on the answers to the following two questions: (1) What kind of new physics is required? (2) Is it allowed by current experimental data?

Weak interactions change a $d$ quark into an $s$ quark via the $W$ boson according to the effective four-quark interaction

$$
\mathcal{L}_{e f f}=\frac{G_{F}}{\sqrt{2}} \sin \theta_{C} \cos \theta_{C} \bar{s} \gamma_{\mu}\left(1-\gamma_{5}\right) u \bar{u} \gamma^{\mu}\left(1-\gamma_{5}\right) d+h . c .
$$

which has the selection rule $\Delta S=1$. This is how two-flavor quark matter may convert into strange matter at sufficiently high densities.

Consider now the effective four-quark operator $(\bar{s} d)^{2}$ which has the selection rule $\Delta \mathrm{S}=2$. Its magnitude is very much suppressed phenomenologically by the smallness of the $K_{L}-K_{S}$ mass difference and is approximately given by $G_{F}^{2} m_{c}^{2} \sin ^{2} \theta_{C} \cos ^{2} \theta_{C} / 16 \pi^{2}$.

What about a $\Delta S=3$ interaction? If it comes from the known weak interaction, it will of course be totally negligible. However, new interactions may generate effective operators such as

$$
\mathcal{L}_{\text {eff }}^{\prime}=\frac{1}{M^{5}}\left(\bar{s}_{R} \bar{s}_{R} \bar{s}_{R}\right)_{8}\left(d_{R} d_{R} d_{R}\right)_{8}+\text { h.c. }
$$

where the subscript 8 refers to the color octet combination of the three quark triplets inside the parentheses. This operator may not be negligible as shown below. 
The effective interaction (5) may cause transitions such as

$$
\Xi^{0}(u s s) \rightarrow n(u d d)+K^{0}(d \bar{s})
$$

but since $1315<940+498$, it is kinematically forbidden. In fact, the only allowed decay from $(5)$ is

$$
\Omega^{-}(s s s) \rightarrow n(u d d)+\pi^{-}(d \bar{u})
$$

where $1672>940+140$. The experimental upper limit on this branching fraction is about $10^{-4}$. [There are only a total of $1.2 \times 10^{4}$ observed $\Omega^{-}$events in the world.] Since the $s$ quarks in $\Omega^{-}$form a color singlet, whereas the operator (5) involves a color octet, two gluons must also be emitted for the proper rearrangement of the color wavefunctions. Hence the amplitude for $\Omega^{-} \rightarrow n \pi^{-}$is proportional to

$$
\frac{\alpha_{S}}{M^{5}}|\psi(0)|^{2} \sim \frac{\alpha_{S}}{M^{5} \pi R^{3}}
$$

where $R \sim 1 \mathrm{fm}$. Comparing this against the dominant decays of $\Omega^{-}$to $\Lambda K^{-}$and $\Xi^{0} \pi^{-}$, the bound

$$
\left(\frac{\alpha_{S}}{M^{5} \pi R^{3} G_{F} \sin \theta_{C} \theta_{C}}\right)^{2}<10^{-4}
$$

can then be obtained. For $\alpha_{S} \sim 0.3$, this implies $M>7.9 \mathrm{GeV}$.

Actually, because the $\Delta \mathrm{S}=2$ interaction is so small, a bound can be obtained also by tying one pair of $d$ and $s$ quarks in (5) to simulate an effective $(\bar{s} d)^{2}$ operator. Hence

$$
\frac{1}{M^{5}} \frac{m_{s} m_{d} m_{c}^{2}}{\left(16 \pi^{2}\right)^{2} M_{W}^{2}} \sin \theta_{C} \cos \theta_{C} m_{K}<\frac{G_{F}^{2} m_{c}^{2} \sin ^{2} \theta_{C} \cos ^{2} \theta_{C}}{16 \pi^{2}}
$$

is required, which implies $M>1.9 \mathrm{GeV}$ for $m_{s}=150 \mathrm{MeV}$ and $m_{d}=10 \mathrm{MeV}$.

The above phenomenological analysis shows that the new physics mass scale is not very much constrained. On the other hand, any specific model for realizing (5) will require the existence of new particles whose interactions with the $d$ and $s$ quarks would be such that 
they should be produced in current high-energy hadron colliders if their masses are of order $100 \mathrm{GeV}$ or less. For example, let there be an exact discrete $Z_{3}$ symmetry so that under $S U(3)_{C} \times U(1)_{Q} \times Z_{3}$,

$$
d_{R} \sim\left(3,-\frac{1}{3}, \omega\right), \quad s_{R} \sim\left(3,-\frac{1}{3}, \omega^{2}\right)
$$

where $\omega^{3}=1$. Add two exotic scalar particles

$$
Q_{d} \sim\left(6,-\frac{2}{3}, \omega^{2}\right), \quad Q_{s} \sim\left(6,-\frac{2}{3}, \omega\right)
$$

and one exotic fermion

$$
\psi_{8} \sim(8,-1,1)
$$

Then the desired interaction (5) is obtained. It should be noted that the above scenario conserves baryon number whereas a similar one proposed earlier does not [11, 12]. See Fig. 2 of Ref. [12]. The proposed exotic particles have strong interactions and should be copiously produced in high-energy hadron colliders, but their detection may be difficult since they may decay only into $d$ and $s$ quarks.

\section{Conclusion and Outlook}

At greater than nuclear densities, matter may convert into a mixture of nucleons, hyperons,

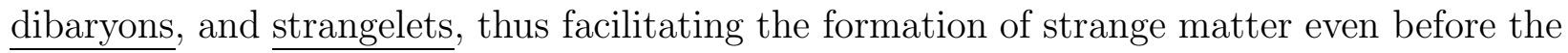
onset of the quark-matter phase transition. The fact that we have very little laboratory information on $\Lambda \Lambda$ interactions does not preclude an important role for such and other pro-

cesses inside neutron stars. This should be studied further in the future. On the speculative side, it may even be possible to start from a nonstrange dibaryon condensate and leapfrog into strange matter with the six-quark effective interaction $(\bar{s} \bar{s} \bar{s})_{8}(d d d)_{8}$, whose magnitude is not very much phenomenologically constrained. 


\section{ACKNOWLEDGEMENT}

I thank the organizers of SQM97 for their great hospitality. This work was supported in part by the U. S. Department of Energy under Grant No. DE-FG03-94ER40837.

\section{References}

[1] E. Witten, Phys. Rev. D30 (1984) 272.

[2] S. Chin and A. Kerman, Phys. Rev. Lett. 43 (1979) 1292.

[3] K. S. Cheng and Z. G. Dai, Phys. Rev. Lett. 77 (1996) 1210.

[4] N. K. Glendenning, Phys. Lett. 114B (1982) 392.

[5] M. Prakash, S. Reddy, J. M. Lattimer, and P. J. Ellis, Heavy Ion Physics 4 (1996) 271.

[6] J. Schaffner, J. Bondorf, and I. N. Mishustin, Heavy Ion Physics 4 (1996) 293.

[7] A. Faessler, A. J. Buchmann, M. I. Krivoruchenko, and B. V. Martemyanov, Phys. Lett. B391 (1997) 255.

[8] R. L. Jaffe, Phys. Rev. Lett. 38 (1977) 195.

[9] F. C. Michel, Nucl. Phys. B (Proc. Suppl.) 24B (1991) 33.

[10] J. Madsen, in Strangeness in Hadronic Matter ( $S$ '95), ed. by J. Rafelski (American Institute of Physics, New York, 1995), p.32.

[11] E. Keith and E. Ma, Heavy Ion Physics 4 (1996) 381.

[12] E. Keith and E. Ma, Mod. Phys. Lett. A12 (1997) 697. 